PALABRAS CLAVE

Mujeres

Empleo de la mujer

Mercado de trabajo

Discriminación basada en el género

Salarios

Encuestas de hogares

Pobreza

Distribución del ingreso

Brasil
Rosycler Cristina Santos Simão

Candidata al doctorado,

Universidad Estadual de Campinas

- rosycler@eco.unicamp.br

Sandro Eduardo Monsueto

Candidato al doctorado,

Universidad Autónoma de Madrid

œ sandro.monsueto@estudiante.uam.es
REVISTA DE LA CEPAL 95 AGOSTO 2008

\section{Impactos de la}

discriminación por género

sobre la pobreza brasileña

\author{
Rosycler Cristal Santos Simão y Sandro Eduardo Monsueto
}

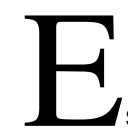

ste trabajo analiza los efectos de la discriminación por género sobre la pobreza brasileña entre 1992-2001, con datos tomados de la Encuesta nacional de hogares. Se estimó una distribución contrafactual del ingreso familiar per cápita bajo la hipótesis de que el mercado de trabajo paga iguales salarios a hombres y mujeres según sus atributos productivos. Los resultados muestran que al eliminar la discriminación de género en el mercado de trabajo, el porcentaje de personas pobres tiende a disminuir, en promedio, un 10\%. Este impacto es aún más significativo entre los grupos poblacionales más vulnerables, como por ejemplo los residentes en hogares encabezados por mujeres negras, sin contrato formal de trabajo o sin afiliación sindical. 


\section{I}

\section{Introducción}

El problema de la pobreza ha estado entre las preocupaciones permanentes de Brasil y de América Latina durante las tres últimas décadas, y ha dado origen a una variada literatura sobre el tema. En líneas generales, respecto de los factores condicionantes de la pobreza en Brasil y en los demás países de la región, los investigadores coinciden en dos aseveraciones: i) la pobreza en Brasil no es un problema de escasez de recursos, sino función de la enorme desigualdad en la distribución del ingreso, y ii) una proporción importante de esta desigualdad se gesta en el mercado de trabajo.

Los datos del Panorama Social de América Latina (CEPAL, 2005) revelan que en el 2002 cerca de $44 \%$ de la población latinoamericana estaba clasificada como pobre y un 19,4\% como indigente; esto quiere decir que se hallaban en situación de pobreza y de indigencia, respectivamente, 221 millones de personas y 97 millones de personas. Respecto a Brasil, en Barros, Henriques y Mendonça (2001) se muestra que entre 1977-1999 el comportamiento de la pobreza fue de relativa estabilidad, ya que el porcentaje de pobres osciló entre $40 \%$ y $45 \%$. Según los autores citados, la extrema desigualdad en la distribución del ingreso laboral en Brasil es la principal causante de la pobreza, puesto que en comparaciones internacionales se comprueba que en países con un ingreso per cápita similar al brasileño la proporción de pobres es inferior al promedio de Brasil. Esto indica hasta qué punto pesa la pésima distribución del ingreso en la magnitud de la pobreza nacional.

En este contexto, las investigaciones sobre la pobreza y la desigualdad han apuntado a identificar qué grupos sociales y demográficos presentan los problemas más críticos, y han comprobado que, en la región, las mujeres constituyen uno de los grupos más vulnerables. Trabajos como los de Oakley (1972), Kabeer (1998) y Montaño (2003) muestran que la pobreza y la exclusión social son problemas que afectan de manera diferenciada a hombres y mujeres y que una de las causas de esta diferencia es el modo como funciona el mercado de trabajo, en el que campean la segmentación y la discriminación por razones de género. Como destacan Costa, Pinheiros y otros (2005), los factores que determinan la mayor vulnerabilidad de las mujeres son esencialmente: i) la desigualdad de la participación en el mercado de trabajo; ii) la poca valoración económica y social de las tareas desempeñadas por mujeres, traducida en salarios más bajos y una segregación ocupacional que restringe a las mujeres a los puestos de trabajo más precarios y menos formalizados; iii) la desigualdad de acceso a recursos productivos como el crédito, la tierra y otros tipos de capital productivo, y iv) la desigualdad de oportunidades para acceder a niveles decisorios, ilustrada por la baja participación femenina en puestos de mando tanto en el sector público como en el privado.

Esto pone en evidencia que la discriminación por género en los distintos espacios de la sociedad acentúa el nivel de pobreza de esa sociedad (Valenzuela, 2003). Siendo el mercado laboral la más importante fuente de ingresos de la población, es razonable suponer que la discriminación salarial que afecta a las mujeres esté altamente correlacionada con la proporción de personas pobres en el país, sobre todo en hogares en los que las mujeres son las principales proveedoras del sustento familiar, fenómeno cada vez más visible en la sociedad brasileña.

En el presente artículo se busca delinear algunas características de la influencia de un mercado laboral discriminatorio por género sobre el nivel de pobreza y desigualdad en el Brasil metropolitano de los años 1990. Con este objetivo, se lleva a cabo un ejercicio de microsimulación con datos de la Encuesta nacional de hogares del país, ${ }^{1}$ para crear un escenario de ingreso per cápita en el cual el mercado de trabajo no discrimine a las mujeres y observar el comportamiento de los indicadores de pobreza en virtud de esta nueva distribución del ingreso.

Después de esta introducción, en la sección II se analiza brevemente la relación entre el mercado de trabajo femenino y la pobreza. En la sección III se desarrolla un modelo simple de microsimulación para calcular el ingreso per cápita en un mercado de trabajo que no discrimina por razones de género. Los resultados se presentan y analizan en las secciones IV y V. En la sección VI se ofrecen algunas consideraciones finales.

\footnotetext{
${ }^{1}$ Pesquisa Nacional por Amostra de Domicilios (PNAD).
} 


\section{II}

\section{Género, mercado de trabajo y pobreza}

Los resultados de investigaciones anteriores muestran que la mayor vulnerabilidad de la mujer a la pobreza está fuertemente relacionada con una inserción precaria en el mercado laboral, el desempeño en actividades de bajo salario, sobre todo en el sector informal, y un más difícil acceso a recursos productivos como el conocimiento y la educación (Valenzuela, 2003; Arriagada, 2005). En esta sección se ponen de relieve algunos de los elementos presentes en esas investigaciones y se entregan también algunos datos relativos al caso de Brasil.

Autores como Bardhan y Udry (1999) señalan algunos hechos que condicionan la entrada de las mujeres en el mercado de trabajo o en actividades del sector productivo de América Latina. Uno es la falta de servicios de atención a la infancia - la escasez de guarderías públicas, por ejemplo- que con frecuencia lleva a las hijas de mujeres trabajadoras a abandonar la escuela para cuidar a sus hermanos menores, perpetuando así el ciclo de baja instrucción en la población femenina más pobre. Otro es la presencia de barreras legales o culturales, que limitan la incorporación de las mujeres al mercado de trabajo. Un tercero — que la investigación destaca- es que los compromisos domésticos pueden restringir severamente la movilidad de las mujeres y sus posibilidades de buscar mejores oportunidades de trabajo. Y un cuarto es que, además, en muchos casos las mujeres son excluidas de programas de crédito, porque no están en condiciones de ofrecer garantías patrimoniales.

Vemos así que la entrada en las actividades productivas está más limitada para las mujeres que para los varones. En Brasil, los datos de la Encuesta nacional de hogares muestran que en las regiones metropolitanas y durante los años 1990, las mujeres tuvieron una menor participación que los hombres en el mercado de trabajo y una mayor incidencia en el desempleo. En promedio, el desempleo entre los varones fue de $8 \%$, mientras que el de las mujeres llegó a 12\%, lo que refleja su menor capacidad de generar ingreso.

Una vez incorporadas al mercado de trabajo, las mujeres enfrentan un segundo conjunto de barreras que restringe su acceso a puestos mejor pagados. Cabía esperar que el aumento de la participación de las mujeres trajera un significativo descenso de la segmentación y de la discriminación salarial. No fue así, sin embargo, ya que no disminuyeron las desigualdades profesionales ni la discriminación salarial por razones de género. Según Arriagada (2005), ninguno de los países latinoamericanos ha logrado igualar el salario de hombres y mujeres, siendo persistente la segregación ocupacional que dificulta a las mujeres el acceso a los mismos puestos de trabajo que los hombres y a remuneraciones concordantes con su calificación. Hoffmann (2000) comprueba, después de considerar una serie de variables, que en 1997 el salario medio de las mujeres era un 33,9\% más bajo que el de los varones. Hoffmann y Leone (2004) encuentran que en 1981 la remuneración laboral de las mujeres equivalía a un $55,7 \%$ del salario de los hombres y que venía mostrando una débil tendencia a mejorar, pasando a representar un 70,6\% del salario masculino en el 2002.

Según datos de la Encuesta nacional de hogares, en el año 2001 el 27\% de las mujeres ocupadas en las regiones metropolitanas de Brasil estaban empleadas sin carteira de trabalho ${ }^{2}$ (el documento laboral estándar) en actividades altamente relacionadas con el segmento informal y de salarios medios inferiores. Entre los varones, en el mismo año, la tasa era de $18 \%$, lo que refleja la asignación de mejores ocupaciones a los varones.

La distribución de las mujeres en el mercado de trabajo y la discriminación salarial seguramente tienen un importante impacto en el ingreso de las familias. Como muestra el cuadro 1, en las zonas metropolitanas de Brasil la contribución del salario femenino al ingreso familiar viene presentando una ligera tendencia al aumento, pero todavía es inferior a la mitad de la contribución del salario masculino. El aporte más bajo de las mujeres revela una mayor dificultad para incorporarse al mercado de trabajo y su inserción en ocupaciones precarias, y lleva a preguntarse cuánto influye el ingreso inferior de las mujeres en la magnitud de la pobreza en el país.

\footnotetext{
${ }^{2}$ La Carteira deTrabalho e Previdência Social, establecida en Brasil en 1932, es un documento que acredita la vida laboral del trabajador y le garantiza el acceso a algunos de los principales derechos laborales, como las prestaciones por desempleo, los beneficios de protección social y los programas de apoyo al trabajador. En general, el documento es una suerte de contrato de trabajo estándar, siendo obligatorio en la mayor parte de las relaciones de trabajo del país (excepto para los funcionarios públicos y militares) y considerándose trabajador formal todo aquel del sector privado que esté trabajando al amparo de este documento. Sin embargo, con la legalización y difusión de contratos de trabajo atípicos y sobre todo con la ley de cooperativas de trabajo de 1994 , la separación entre los sectores formal e informal basada únicamente en la carteira de trabalho se ha tornado más complicada.
} 


\begin{tabular}{|c|c|c|c|c|}
\hline \multirow{2}{*}{ Año } & \multirow{2}{*}{$\begin{array}{l}\text { Promedios } \\
\text { (dólares) }\end{array}$} & \multicolumn{3}{|c|}{$\begin{array}{l}\text { Participación en el ingreso familiar del ingreso proveniente del trabajo principal } \\
\text { (En porcentajes) }\end{array}$} \\
\hline & & Total & Varones & Mujeres \\
\hline 1992 & 147,7 & 79,2 & 57,3 & 21,9 \\
\hline 1993 & 165,4 & 78,4 & 57,0 & 21,4 \\
\hline 1995 & 197,8 & 78,6 & 56,4 & 22,3 \\
\hline 1996 & 203,6 & 77,9 & 54,6 & 23,2 \\
\hline 1997 & 198,4 & 78,3 & 54,8 & 23,5 \\
\hline 1998 & 204,5 & 76,1 & 52,9 & 23,1 \\
\hline 1999 & 183,7 & 75,3 & 51,4 & 23,9 \\
\hline 2001 & 186,2 & 75,5 & 50,6 & 24,8 \\
\hline Promedio & 185,9 & 77,4 & 54,4 & 23,0 \\
\hline
\end{tabular}

Fuente: elaboración propia a partir de datos de la Encuesta nacional de hogares.

En síntesis, el mercado de trabajo latinoamericano y, en especial, el brasileño exhiben una marcada segregación y diferencias salariales por razones de género que pueden afectar severamente el nivel de ingreso per cápita de la sociedad. En las secciones siguientes se presenta un ejercicio econométrico que puede contribuir a que se entienda mejor la relación entre la discriminación y el nivel de pobreza en el país.

\section{III}

\section{Metodología y fuente de los datos}

Las estimaciones de esta investigación siguen las líneas del enfoque utilizado por Gradín, Río y Cantó (2006) para analizar mediante microsimulaciones el efecto de la discriminación por género sobre los niveles de pobreza y desigualdad de 12 países de la Unión Europea. La estrategia es, básicamente, la de comparar dos tipos de escenarios de distribución del ingreso familiar per cápita, siendo el primero representativo de un mercado de trabajo real, con discriminación por género (escenario estimado) y el segundo un escenario hipotético en el cual el mercado de trabajo remunera de igual forma a hombres y mujeres conforme a sus atributos productivos.

Considérese que el ingreso familiar per cápita $d$ está formado por la suma del ingreso generado en el mercado de trabajo $(w)$ y del ingreso que proviene de otras fuentes $(\pi)$, dividida por el número de moradores $(n)$ :

$$
d=\frac{\sum_{i}\left(w h_{i} \cdot t_{i}+\pi_{i}\right)}{n}
$$

donde $w h_{i}$ es el salario por hora y $t_{i}$ son las horas trabajadas. En este escenario estimado, el ingreso proveniente del mercado de trabajo está marcado por una diferencia salarial que se debe a la discriminación por razones de género. ${ }^{3}$ Esto significa que la estimación de ecuaciones de salarios separadas para hombres $m$ y para mujeres $f$ debe obtener retornos marginales distintos según las características productivas y personales de cada grupo:

$$
\begin{gathered}
\ln \left(w h_{m}\right)=y_{m}=Z_{m}{ }^{\prime} \beta_{m}+\mu_{, m} \\
\ln \left(w h_{f}\right)=y_{f}=Z_{f}^{\prime} \beta_{f}+\mu_{, f}
\end{gathered}
$$

donde $y_{m}$ y $y_{f}$ representan el logaritmo del salario por hora $(w h)$ de hombres y mujeres, respectivamente; $Z_{i}$ es un conjunto de atributos personales, regionales y del

\footnotetext{
${ }^{3}$ Observe que, por simplificación, no se considera la posibilidad de que el componente del ingreso que no proviene del mercado de trabajo también se encuentre sujeto a discriminación (Gradín, Río y Cantó, 2006).
} 
mercado de trabajo; $\beta_{i}$ es el vector estimado de coeficientes, o retornos marginales, y $\mu_{i}$ es el vector de errores. Para una determinada familia, el ingreso proveniente del mercado de trabajo es representada por:

$$
w h=\exp \left(y_{m}\right)+\exp \left(y_{f}\right)=w h_{m}+w h_{f}
$$

La construcción o simulación del segundo escenario de distribución del ingreso familiar per cápita —el escenario contrafactual, en el cual la discriminación en el mercado de trabajo es teóricamente eliminada y los retornos marginales masculinos y femeninos son igualmente determinados - se basa en la hipótesis de que la diferencia entre $\beta_{m}$ y $\beta_{f}$ es nula. En general, se admite que los retornos marginales que el mercado de trabajo paga a los trabajadores varones son una buena aproximación de la distribución deseada de los salarios en ausencia de discriminación, siguiendo la idea tradicional de autores como Oaxaca (1973), Blinder (1973) o Juhn, Murphy y Pierce (1993). De este modo, el escenario contrafactual puede ser construido con la suposición de que el salario por hora trabajada de las mujeres tiene el mismo vector de retornos marginales que los varones, es decir:

$$
\begin{gathered}
\ln \left(w h_{f}^{*}\right)=y{ }_{f}^{*}=Z_{f}{ }^{\prime} \beta_{m}+\mu_{, f} \\
w h^{*}=\exp \left(y_{m}\right)+\exp \left(y_{f}^{*}\right)=w h_{m}+w h_{f}^{*}
\end{gathered}
$$

y el ingreso familiar per cápita "contrafactual", hipotéticamente libre de discriminación, es determinado por:

$$
d^{*}=\frac{\sum_{i}\left(w h{ }_{i}^{*} \cdot t_{i}+\lambda_{i}\right)}{n}
$$

Finalmente, a partir de las dos distribuciones del ingreso familiar per cápita construidas, la estimada $d$ y la contrafactual $d^{*}$, es posible analizar el impacto de la discriminación a través de la diferencia entre los indicadores de desigualdad y de pobreza de cada escenario. Utilizando un indicador $I$ cualquiera, el impacto de la discriminación por razones de género sobre dicho indicador es dado por:

$$
\Delta I\left(d, d^{*}, l p\right) \%=\frac{I\left(d^{*}, p l\right)-I(d, p l)}{I(d, p l)} * 100
$$

donde $p l$ es la línea de pobreza, usada cuando es necesario. En esta investigación se utilizan un indicador de desigualdad y tres de pobreza: el índice de Gini $(G)$; el porcentaje de personas por debajo de la línea de pobreza $H$; la intensidad de la pobreza $P$-también conocida como razón de insuficiencia de ingreso, que capta en cuánto debería aumentar el ingreso de una persona pobre para que tenga un ingreso igual al umbral de pobreza- $-y$ el índice de Foster, Greer y Thorbecke.

Sin embargo, la existencia de un retorno marginal equiparable entre hombres y mujeres debe de tener un efecto en la oferta y en la demanda de mano de obra femenina, ya que el salario del mercado sin discriminación es más alto para las mujeres. Por un lado, eso aumenta el incentivo a la participación femenina en el mercado de trabajo pero, por otro lado, las empresas pueden responder al alza de los salarios con una merma del nivel de empleo. Admitiendo, para simplificar, que la oferta y la demanda de trabajo masculinas no se ven alteradas, la ecuación de salario contrafactual necesita captar también las nuevas motivaciones de la oferta de mano de obra entre las mujeres.

Algunos trabajos de microsimulación, como el de Bourguignon, Ferreira y Lustig (1998) y el de Yáñez (2004) incorporan esta alteración en un modelo de dos etapas y con una ecuación de selección del mercado de trabajo, componente que es denominado efecto participación o efecto selección. Para el presente ejercicio, el punto de partida puede ser una ecuación de selección del mercado de trabajo que considera la probabilidad de que un individuo esté en una de las siguientes situaciones: 1) está trabajando; 2) está desempleado, y 3) está fuera de la población económicamente activa (PEA). El método econométrico utilizado es una expansión del modelo de Heckman (1979) de sesgo de selección aplicada a múltiples resultados, añadiendo la razón inversa de Mills en las ecuaciones de salario de hombres y mujeres. ${ }^{4} \mathrm{La}$ ecuación de probabilidad de participación en el mercado de trabajo utiliza la población en edad activa, entre los 16 y los 65 años, y está determinada mediante un modelo probit multinomial, con la siguiente forma funcional:

$$
\begin{aligned}
P\left(p_{i}=a\right)= & f\left(\text { edad }, \sum \text { color }, \text { jefe, educación },\right. \\
& \left.\sum \text { región, } \sum \text { año }\right)
\end{aligned}
$$

donde $P\left(p_{i}=a\right)$ es la probabilidad de que una variable $p_{i}$ asuma los valores 1 (trabaja), 2 (desempleado) y 3 (fuera de la PEA); edad es la edad continua del trabajador; color, un conjunto de tres variables ficticias para el color del

\footnotetext{
${ }^{4}$ Se ha utilizado el método propuesto por Bourguignon, Fournier y Gurgand (2007).
} 
individuo (blancos, negros y pardos); ${ }^{5}$ jefe, una variable ficticia con valor 1 para los individuos que son jefes de hogar y 0 en el caso contrario; educación, la escolaridad del trabajador en años de estudio; región, seis variables ficticias, cada una de las cuales representa una región metropolitana, y año, un conjunto de variables ficticias para los años investigados.

Las ecuaciones de salario utilizan la población económicamente activa ocupada en la semana de referencia de la encuesta, también entre 16 y 65 años y con salario y horas trabajadas positivas, estimadas con la siguiente forma funcional:

$$
\begin{gathered}
y_{i}=f\left(\text { edad }, \text { edad }^{2}, \sum \text { color }, \text { jefe, educación, sindicato },\right. \\
\left.\sum \text { status, } \sum \text { actividad }, \sum \text { región }+\sum a \tilde{n} o+\sum \lambda\right)
\end{gathered}
$$

donde edad, color, jefe, educación, región y año son definidas como antes, con el término cuadrado de la edad añadido; sindicato es una variable ficticia para captar si el trabajador está o no afiliado a algún sindicato; status es un conjunto de cuatro variables ficticias para el régimen de trabajo (con carteira de trabalho, sin carteira, funcionario público o militar, autónomo y empleador), actividad es una serie de cuatro variables ficticias para el sector de actividad (industria y construcción, comercio, servicios y otras actividades), y el vector $\lambda$ corresponde a las respectivas razones inversas de Mills, que actúan como factores de corrección del sesgo de selección del mercado de trabajo, obtenidas de la ecuación de participación.

Enseguida, los datos se agregan para obtener el ingreso familiar per cápita estimado (ecuación 1) y el contrafactual (ecuación 7). La fuente de datos empleada es la Encuesta nacional de hogares que realiza anualmente el Instituto Brasileño de Geografía y Estadística (IBGE), excepto en los años en que se han efectuado censos demográficos y el año 1994. El análisis cubre

\footnotetext{
${ }^{5}$ No fueron consideradas las personas que se declararon Amarillas o Indígenas o que no contestaron.
}

el período $1992-2001^{6}$ y abarca personas residentes en seis regiones metropolitanas del país. ${ }^{7}$

Como ingreso laboral se ha considerado exclusivamente el que proviene del trabajo principal del individuo. Otros ingresos son los provenientes de trabajos secundarios, pensiones por jubilación, pensiones por otras causas, abono de trabajo, ${ }^{8}$ alquileres, donaciones y operaciones financieras. Solo se utilizaron hogares particulares permanentes, excluyendo del número de moradores a los pensionistas, los empleados domésticos y los familiares de los empleados domésticos. No fueron considerados los hogares con ingreso total igual a cero.

Para calcular los indicadores fue necesario establecer una línea de pobreza, la que definiría la renta mínima necesaria para la manutención mensual de una persona media. Dada la heterogeneidad de definiciones de líneas de pobreza, ${ }^{9}$ la investigación usó el costo mensual de una cesta básica de alimentos, calculada por el Departamento Intersindical de Estadística y Estudios Socioeconómicos a través de una encuesta mensual sobre el precio de una lista de bienes de consumo (DIEESE, 1993). Se utilizaron los valores de los meses de septiembre de cada año (mes de aplicación de la Encuesta nacional de hogares) y de cada región metropolitana. De esta forma, la línea de pobreza nacional fue definida como el promedio de los costos de las cestas básicas regionales.

Los valores monetarios fueron deflactados —según lo recomendado por Corseuil y Foguel (2002) — a enero del 2002 y convertidos a dólares del mismo mes. El cuadro 2 muestra el número de observaciones, el salario mínimo y la línea de pobreza utilizada en cada año. Los resultados del ejercicio se presentan y analizan en las secciones siguientes.

\footnotetext{
${ }^{6}$ En el 2000 se realizó un censo demográfico, de modo que no se llevó cabo la Encuesta nacional de hogares.

${ }^{7}$ Belo Horizonte (estado de Minas Gerais), Porto Alegre (Rio Grande do Sul), Recife (Pernambuco), Rio de Janeiro (Rio de Janeiro), Salvador (Bahía) y São Paulo (São Paulo).

${ }^{8}$ Beneficio social de un salario mínimo anual asegurados a los trabajadores que hayan cobrado hasta dos salarios mínimos de empleadores afiliados al Programa de Integración Social (PIS) o al Programa de Formación del Patrimonio del Servidor Público (PASEP), creados para fomentar una mejor distribución del ingreso en el país.

${ }^{9}$ Véase un examen detallado del tema en Rocha (2000a).
} 
Brasil: número de observaciones, población en edad activa, población económicamente activa y ocupada, salario mínimo y línea de pobreza, 1992-2001

\begin{tabular}{lcccccc}
\hline Año & $\begin{array}{c}\mathrm{N}^{\mathbf{o}} \text { de } \\
\text { observaciones }\end{array}$ & $\begin{array}{c}\text { Población en } \\
\text { edad activa (\%) }\end{array}$ & $\begin{array}{c}\text { Pob. económicamente } \\
\text { activa (\%) }\end{array}$ & $\begin{array}{c}\text { Población } \\
\text { ocupada (\%) }\end{array}$ & $\begin{array}{c}\text { Salario mínimo } \\
\text { (dólares) }\end{array}$ & $\begin{array}{c}\text { Línea de pobreza } \\
\text { (dólares) }\end{array}$ \\
\hline 1992 & 81757 & 61,7 & 40,9 & 36,8 & 83,4 & 46,2 \\
1993 & 84052 & 61,7 & 41,4 & 37,2 & 70,1 & 43,2 \\
1995 & 86323 & 62,9 & 42,5 & 38,9 & 62,7 & 47,4 \\
1996 & 85683 & 63,6 & 42,7 & 38,4 & 62,4 & 46,9 \\
1997 & 89004 & 64,0 & 43,2 & 38,1 & 64,1 & 43,8 \\
1998 & 89487 & 64,6 & 43,8 & 37,7 & 67,4 & 46,9 \\
1999 & 92087 & 64,8 & 44,3 & 37,7 & 66,2 & 46,4 \\
2001 & 90717 & 65,6 & 45,5 & 39,5 & 76,3 & 47,3 \\
Total & 699110 & 63,7 & 43,1 & 38,1 & - & - \\
\hline
\end{tabular}

Fuente: Encuesta nacional de hogares e Instituto de Investigación Económica Aplicada (IPEA).

\section{IV}

\section{El impacto sobre la desigualdad}

El efecto de la discriminación por razones de género sobre la desigualdad y la pobreza se analiza mediante la comparación de la diferencia de salarios entre hombres y mujeres en mercados de trabajo con y sin discriminación por razones de género. Las ecuaciones de participación en el mercado de trabajo y las de salario obtienen signos esperados y significativos en la mayoría de los casos (véase el apéndice). Los varones presentan efectos marginales superiores a los que exhiben las mujeres en variables importantes como la educación o la tenencia de un puesto de trabajo formal, lo que indica la presencia de un impacto diferenciado sobre la determinación de los salarios.

El gráfico 1 exhibe la evolución de la diferencia de ingreso por hora entre hombres y mujeres en el trabajo principal, tanto observada como estimada y contrafactual. Se verifica que, incluso con la sistemática disminución de la brecha salarial, las mujeres ganan aproximadamente un 27\% menos que los varones. Comparando la diferencia estimada con la contrafactual se concluye que si el mercado de trabajo remunerara de la misma manera a hombres y a mujeres - es decir, conforme a sus atributos productivos y la distribución ocupacional- no solo sería menor la diferencia de salario sino que además la tendencia a disminuir sería más significativa durante el periodo de análisis. En promedio, un mercado sin discriminación por razones de género elevaría el salario de las mujeres en $28 \%$ en dicho periodo.

La persistencia de una diferencia de salarios aun después de incorporar el efecto de la discriminación por género se debe en parte a errores de medida, pero también a aspectos vinculados a la distribución de factores productivos, como la edad más elevada de los varones o la mayor concentración de mujeres en actividades de salarios bajos, como el empleo doméstico. A causa de la segregación ocupacional, alrededor del 17\% de las mujeres ocupadas trabajan en actividades domésticas, mientras que entre los varones esta proporción no supera el $2 \%$. Si el contingente de empleados domésticos no se considera en el cálculo de los salarios (gráfico 1, última línea), la diferencia pasa a ser favorable a las mujeres, ya que estas poseen un mayor acervo de otros factores productivos, sobre todo educación. Por largo tiempo las mujeres han alcanzado un promedio de años de estudio superior al de los hombres, pero esto no les ha garantizado una mejor inserción ocupacional ni salarios más altos.

El impacto de esta diferencia salarial sobre la desigualdad en la distribución del ingreso familiar per cápita es analizado en el cuadro 3, utilizando los valores estimados y contrafactuales del ingreso y del índice de concentración. En general, al eliminarse la discriminación por razones de género en el mercado de trabajo el ingreso per cápita aumenta $6,8 \%$ y el coeficiente de Gini disminuye $0,7 \%$ en promedio. La diferencia entre el ingreso per cápita estimado y el contrafactual también puede ser entendida como la compensación necesaria para que el efecto de la discriminación salarial sea anulado, o bien como el costo social de la diferenciación por razones de género. 
GRÁFICO 1

Brasil: evolución de la diferencia de salario por hora entre hombres y mujeres, observada, estimada, y contrafactual con y sin trabajadores domésticos, 1992-2001 (Porcentajes)

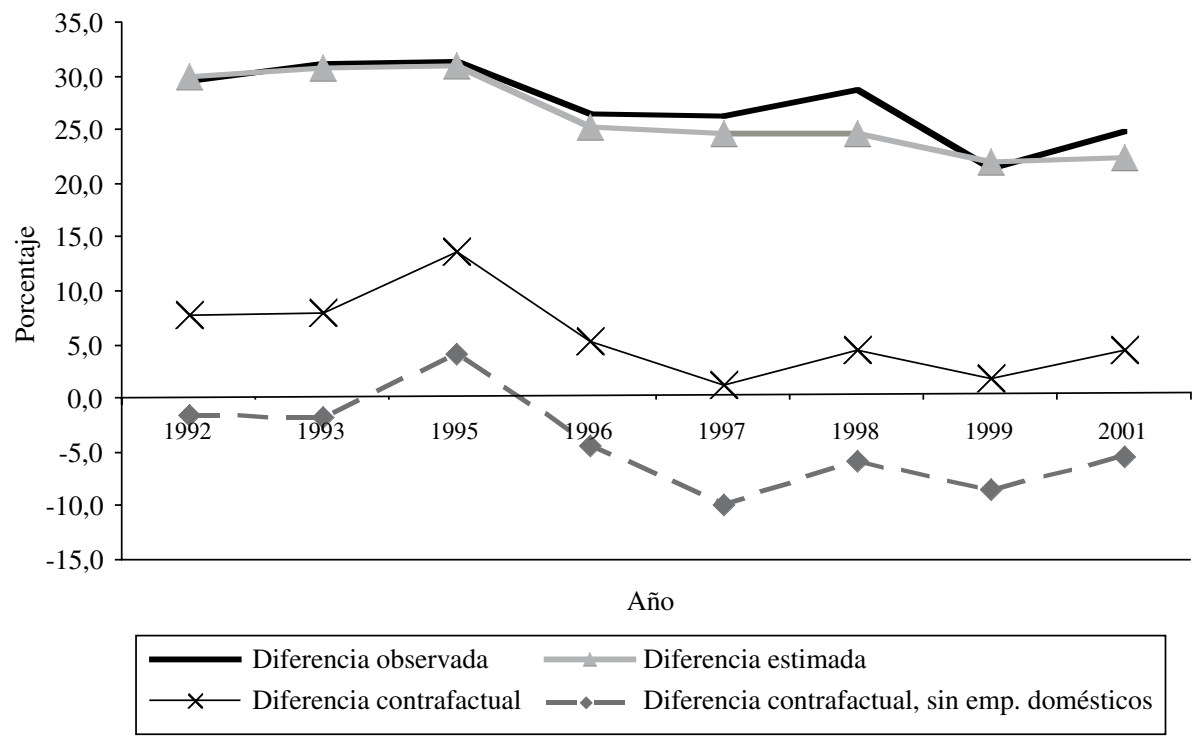

Fuente: elaboración propia a partir de datos de la Encuesta nacional de hogares.

CUADRO 3

Brasil: impacto de la discriminación salarial por género sobre el ingreso familiar per cápita y sobre la desigualdad, 1992-2001 (Ingreso familiar per cápita y porcentajes)

\begin{tabular}{|c|c|c|c|c|c|c|}
\hline \multirow[b]{2}{*}{ Año } & \multicolumn{3}{|c|}{ Ingreso familiar per cápita (dólares) } & \multicolumn{3}{|c|}{ Coeficiente de Gini } \\
\hline & Estimado & Contrafactual & $\begin{array}{c}\text { Variación } \\
(\%)\end{array}$ & Estimado & Contrafactual & $\begin{array}{c}\text { Variación } \\
(\%)\end{array}$ \\
\hline 1992 & 126,5 & 135,7 & 7,2 & 0,4875 & 0,4861 & $-0,3$ \\
\hline 1993 & 124,9 & 134,1 & 7,4 & 0,4992 & 0,4958 & $-0,7$ \\
\hline 1995 & 162,6 & 172,4 & 6,0 & 0,4973 & 0,4936 & $-0,7$ \\
\hline 1996 & 169,4 & 180,5 & 6,6 & 0,4926 & 0,4908 & $-0,4$ \\
\hline 1997 & 165,8 & 178,6 & 7,7 & 0,4917 & 0,4880 & $-0,7$ \\
\hline 1998 & 171,0 & 182,4 & 6,6 & 0,5036 & 0,4994 & $-0,8$ \\
\hline 1999 & 153,9 & 163,8 & 6,5 & 0,4977 & 0,4922 & $-1,1$ \\
\hline 2001 & 155,3 & 164,8 & 6,1 & 0,4941 & 0,4883 & $-1,2$ \\
\hline Promedio & 153,7 & 164,0 & 6,8 & 0,4955 & 0,4918 & $-0,7$ \\
\hline
\end{tabular}

Fuente: elaboración propia a partir de datos de la Encuesta nacional de hogares.

El coeficiente de concentración del ingreso (Gini) presenta una marcada tendencia a disminuir entre 1993 y 1997, como efecto directo del brusco descenso de la inflación propiciado por el programa federal de estabilización monetaria en el período. El impacto de la discriminación sobre este indicador tiende a aumentar hasta el final de la década, pese a una disminución de la diferencia salarial. Sin embargo, con el aumento de la participación del salario de la mujer en el ingreso familiar (véase más atrás el cuadro 1), la diferencia de ingreso laboral por razones de género pesa cada vez más en el conjunto de la sociedad, implicando el aumento del impacto absoluto de la discriminación sobre el coeficiente de Gini. 
GRÁFICO 2

Brasil: variación por la discriminación por género, según deciles del ingreso familiar per cápita, 1992, 2001 y período 1992-2001

(Porcentajes)

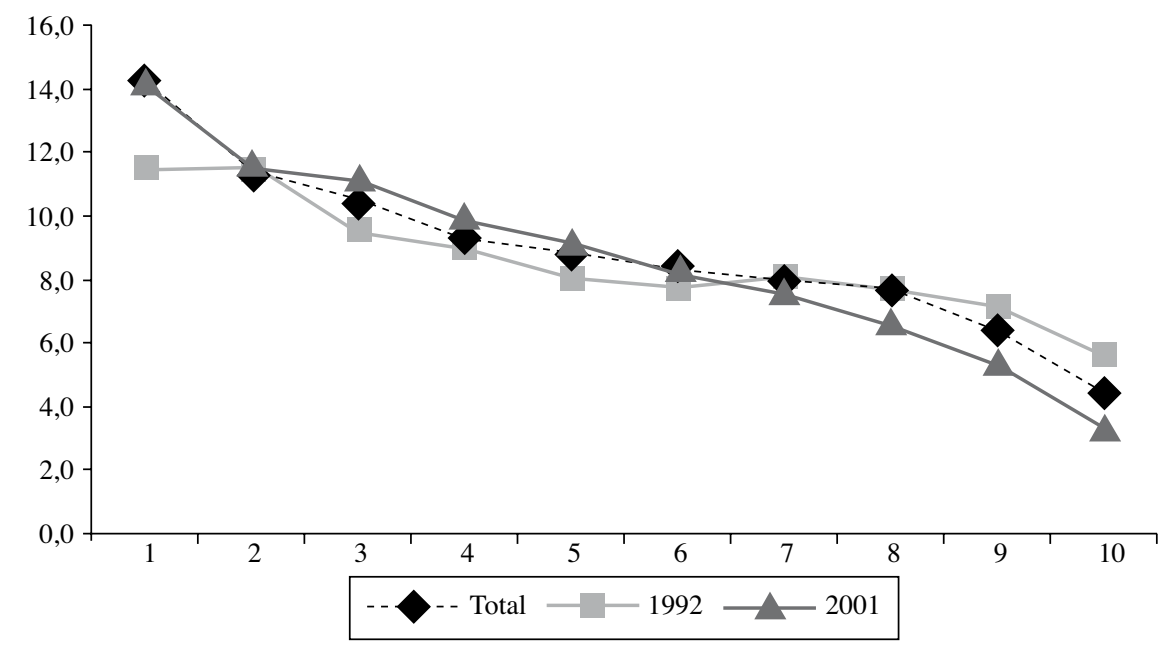

Fuente: elaboración propia a partir de los datos de la Encuesta nacional de hogares.

Gradín, Río y Cantó (2006) observan un efecto distinto de la discriminación sobre la concentración del ingreso en países de la Unión Europea, pues constatan un aumento del coeficiente de Gini. Los autores citados argumentan que esto es consecuencia de que, en el caso europeo, al comparar el efecto de la discriminación en los diversos deciles de ingreso per cápita se observa que ese efecto es mayor en la mediana de la distribución y menor entre los más pobres. De esta forma, los hogares más ricos son beneficiados en mayor medida por la eliminación de la discriminación y por el aumento del salario de las mujeres, concentrando aún más el ingreso. El gráfico 2 reproduce este ejercicio para el caso brasileño y permite observar un comportamiento distinto hasta la mediana de la distribución, decreciente en la medida en que se avanza hacia los hogares más ricos. En otras palabras, el costo social de la discriminación por razones de género en el mercado de trabajo es más alto en los hogares más pobres. Además, comparando las distribuciones en los años extremos del periodo, 1992 y 2001, se comprueba un aumento del impacto sobre los más pobres y una disminución entre los más ricos.

En general, los resultados muestran una mejora en la distribución del ingreso per cápita en función de un posible decrecimiento de la discriminación salarial por razones de género en el mercado de trabajo. En la sección siguiente se evalúa también el efecto de esta discriminación en el nivel de pobreza del país.

\section{Discriminación por razones de género e impacto sobre la pobreza}

El cuadro 4 muestra las estimaciones de los tres indicadores de pobreza utilizados y el impacto sobre ellos de la discriminación por razones de género en el mercado de trabajo. En el período analizado (1992-2001), en promedio, el 21\% de la población del Brasil metropolitano vivía con menos de la mitad de un salario mínimo y la distancia entre el ingreso medio de los pobres y la línea que define la pobreza era de 8 dólares. El nivel 
CUADRO 4

Brasil: efecto sobre la pobreza de la eliminación

de la discriminación salarial por género, 1992-2001

(Porcentajes e indicadores)

\begin{tabular}{|c|c|c|c|c|c|c|c|c|c|}
\hline \multirow{2}{*}{ Año } & \multicolumn{3}{|c|}{ Porcentaje de pobreza } & \multicolumn{3}{|c|}{ Intensidad de la pobreza } & \multicolumn{3}{|c|}{$\begin{array}{c}\text { Índice de Foster, Greer y } \\
\text { Thorbecke }\end{array}$} \\
\hline & Estimado & Contrafactual & $\begin{array}{c}\text { Variación } \\
(\%)\end{array}$ & Estimado & Contra-factual & $\begin{array}{l}\text { Variación } \\
\quad(\%)\end{array}$ & Estimado & Contrafactual & $\begin{array}{l}\text { Variación } \\
\quad(\%)\end{array}$ \\
\hline 1992 & 25,7 & 23,4 & $-9,3$ & 10,1 & 8,9 & $-11,5$ & 15,1 & 13,5 & $-10,6$ \\
\hline 1993 & 24,8 & 22,2 & $-10,4$ & 9,8 & 8,5 & $-12,8$ & 14,6 & 12,9 & $-11,8$ \\
\hline 1995 & 19,6 & 17,6 & $-10,4$ & 7,5 & 6,5 & $-12,1$ & 11,3 & 10,0 & $-11,3$ \\
\hline 1996 & 18,4 & 16,4 & $-10,7$ & 7,1 & 6,2 & $-11,7$ & 10,6 & 9,4 & $-11,3$ \\
\hline 1997 & 17,0 & 14,8 & $-13,0$ & 6,5 & 5,6 & $-14,7$ & 9,9 & 8,5 & $-14,1$ \\
\hline 1998 & 19,8 & 17,7 & $-10,7$ & 7,4 & 6,5 & $-12,5$ & 11,3 & 10,0 & $-11,7$ \\
\hline 1999 & 21,2 & 18,9 & $-10,7$ & 8,2 & 7,2 & $-12,4$ & 12,4 & 10,9 & $-11,7$ \\
\hline 2001 & 21,6 & 19,6 & $-9,3$ & 8,7 & 7,6 & $-12,1$ & 12,9 & 11,5 & $-11,0$ \\
\hline Media & 21,0 & 18,8 & $-10,5$ & 8,2 & 7,1 & $-12,5$ & 12,3 & 10,8 & $-11,7$ \\
\hline
\end{tabular}

Fuente: elaboración propia a partir de los datos de la Encuesta nacional de hogares.

CUADRO 5

Brasil: efecto de la eliminación de la discriminación salarial por género sobre el porcentaje de personas bajo la línea de pobreza, 1992-2001 (Porcentajes)

\begin{tabular}{|c|c|c|c|c|c|c|}
\hline \multirow{2}{*}{ Año } & \multicolumn{2}{|c|}{ Nordeste } & \multicolumn{3}{|c|}{ Sudeste } & \multirow{2}{*}{$\frac{\text { Sur }}{\text { Porto Alegre }}$} \\
\hline & Recife & Salvador & Belo Horizonte & Río de Janeiro & São Paulo & \\
\hline 1992 & $-7,0$ & $-7,8$ & $-9,1$ & $-8,6$ & $-11,6$ & $-8,5$ \\
\hline 1993 & $-7,7$ & $-8,3$ & $-9,9$ & $-9,6$ & $-13,5$ & $-10,3$ \\
\hline 1995 & $-8,1$ & $-9,5$ & $-9,0$ & $-9,6$ & $-14,2$ & $-8,5$ \\
\hline 1996 & $-7,5$ & $-9,3$ & $-13,9$ & $-9,8$ & $-12,8$ & $-10,0$ \\
\hline 1997 & $-10,8$ & $-11,5$ & $-13,3$ & $-10,7$ & $-16,9$ & $-12,9$ \\
\hline 1998 & $-8,3$ & $-11,2$ & $-14,3$ & $-9,1$ & $-11,8$ & $-9,4$ \\
\hline 1999 & $-8,6$ & $-12,0$ & $-11,2$ & $-7,4$ & $-13,9$ & $-10,1$ \\
\hline 2001 & $-9,4$ & $-13,0$ & $-9,8$ & $-6,5$ & $-10,3$ & $-7,7$ \\
\hline Promedio & $-8,4$ & $-10,3$ & $-11,3$ & $-8,9$ & $-13,1$ & $-9,7$ \\
\hline
\end{tabular}

Fuente: elaboración propia a partir de datos de la Encuesta nacional de hogares.

de pobreza disminuyó marcadamente hasta mediados de los años 1990, sobre todo gracias al crecimiento económico y a la estabilidad inflacionaria en el período, que disminuyó significativamente las consecuencias del impuesto inflación sobre los más pobres. Sin embargo, como argumenta Rocha (2000b), pasados los efectos distributivos del plan de estabilización (el Plan Real, aplicado a partir de 1994), aumentaron en el país la incidencia de la pobreza, la intensidad de la pobreza y el índice de Foster, Greer y Thorbecke.

En los años 1990 la discriminación en el mercado de trabajo influyó cada vez más en el nivel de pobreza de la población metropolitana; en promedio, eliminar esa discriminación reduciría en 10,5\% la proporción de personas pobres. Del mismo modo, la evolución del efecto de la discriminación aparece también relacionada con el ritmo de crecimiento económico del país (Barros y Mendonça, 1997; Sobrinho, 2001). Los períodos de bonanza del ciclo económico contribuyen a paliar los efectos de la discriminación sobre la proporción de pobres, debido a las consecuencias distributivas del crecimiento económico.

Uno de los factores que dificultan el análisis de la pobreza en Brasil es su marcada heterogeneidad regional, con núcleos de pobreza que se encuentran en regiones con menor nivel de desarrollo y urbanización y también en regiones industriales como São Paulo. De igual modo, la discriminación también tiene efectos distintos según el nivel de pobreza de cada región (cuadro 5). Utilizando el ingreso familiar per cápita estimado, se encuentra que Recife y Salvador, en la región Nordeste del país, tienen 


\begin{tabular}{llcc}
\hline Año & Total & Mujeres negras & Mujeres sin carteira $a^{\mathrm{a}}$ \\
\hline 1992 & $-27,4$ & $-19,7$ & $-46,2$ \\
1993 & $-29,2$ & $-19,2$ & $-37,0$ \\
1995 & $-31,3$ & $-24,1$ & $-39,7$ \\
1996 & $-27,4$ & $-21,3$ & $-48,2$ \\
1997 & $-31,5$ & $-21,4$ & $-48,8$ \\
1998 & $-26,6$ & $-20,5$ & $-44,4$ \\
1999 & $-26,6$ & $-20,6$ & $-44,3$ \\
2001 & $-21,9$ & $-14,6$ & $-32,3$ \\
Media & $-27,7$ & $-20,2$ & $-71,0$ \\
\hline
\end{tabular}

Fuente: elaboración propia a partir de datos de la Encuesta nacional de hogares.

a Carteira: documento de trabajo estándar.

un promedio de $42 \%$ de su población metropolitana por debajo de la línea de pobreza; allí la eliminación de la discriminación por razones de género debería reducir este indicador, en promedio, un $9 \% .{ }^{10}$

El uso del valor de la cesta básica como línea de pobreza es también un indicador del costo de vida. Los resultados del análisis muestran que en regiones donde el costo de vida es más elevado, como São Paulo y Río de Janeiro, un aumento del ingreso generado por un descenso de la discriminación contribuye más a disminuir la pobreza. En cambio, en la región Sur del país, aquí representada por la zona metropolitana de Porto Alegre, que ostenta los mejores indicadores sociales del país —el de desarrollo humano, por ejemplo- el impacto de la diferenciación salarial tiende a ser inferior.

Además de su heterogeneidad regional, la pobreza presenta un complejo perfil en materia de características ocupacionales (Sobrinho, 2001). Entre los hogares pobres, una proporción significativa está encabezada por personas de raza negra, de baja escolaridad y que se desempeñan en ocupaciones con poca protección institucional y salarios bajos, principalmente en actividades agrícolas o de construcción civil. Dada la alta concentración de

\footnotetext{
${ }^{10}$ Véase en el apéndice el porcentaje de personas pobres en cada
} región y las líneas regionales de pobreza. mujeres en ocupaciones precarias y de bajos salarios, las diferencias de ingreso laboral por razones de género, que persisten aun después de considerar las características de los puestos de trabajo, son un elemento más de fomento de la pobreza brasileña.

El cuadro 6 selecciona algunas de esas características relacionadas con las familias más pobres y estima el efecto de la discriminación por género en el porcentaje de personas pobres que residen en hogares encabezados por mujeres, según raza e inserción laboral. En comparación con el cuadro 4, esta nueva información pone en evidencia que al eliminar la discriminación por género en el mercado de trabajo se tiende a beneficiar en mayor medida a las personas en condiciones más precarias. Este resultado destaca la necesidad de medidas de focalización de las políticas públicas encaminadas a aminorar la pobreza en la población brasileña.

En síntesis, la disminución de las diferencias de salario entre hombres y mujeres en el mercado de trabajo, asociada a políticas que promuevan la igualdad de condiciones de acceso a ese mercado, tiende a beneficiar a grupos tradicionalmente más vulnerables en las zonas metropolitanas brasileñas. El crecimiento económico es un instrumento valioso para combatir la pobreza, pero no es suficiente si los logros no se distribuyen parejamente. Son necesarias entonces políticas capaces de focalizar el problema. 


\section{VI}

\section{Consideraciones finales}

Este trabajo ha investigado qué efecto tiene la discriminación por género en el mercado de trabajo sobre el nivel de pobreza en zonas metropolitanas de Brasil, utilizando datos de la Encuesta nacional de hogares para el período 1992-2001 y una línea de pobreza definida por la mitad de un salario mínimo.

Aplicando ecuaciones para calcular la diferencia salarial entre hombres y mujeres, se ha encontrado que la eliminación de la discriminación por género se traduciría en un aumento medio de $28 \%$ del salario por hora de las mujeres en el período investigado y una disminución inferior al $1 \%$ en el coeficiente de concentración del ingreso familiar per cápita. Además, la discriminación tiene como efecto acentuar el nivel de pobreza del país. La eliminación de la discriminación en el mercado de trabajo tiende a disminuir el porcentaje de personas pobres, en promedio, en un 10,5\%. Este valor, bastante pequeño, es más significativo entre los grupos poblacionales de mayor vulnerabilidad, como los integrantes de hogares encabezados por mujeres negras, sin el contrato formal de la carteira de trabalho o sin afiliación sindical.

De esta forma, los resultados apuntan a la necesidad de focalizar las políticas nacionales destinadas a combatir la pobreza. Además, estas políticas deben hacerse extensivas al mercado de trabajo con miras a reducir las diferencias salariales entre hombres y mujeres. Por último, las evidencias presentadas en este trabajo pueden contribuir a fundamentar la defensa de políticas públicas de igualdad de género pues, pese a los avances significativos logrados en la disminución de la desigualdad, todavía queda un largo camino por recorrer. 
APÉNDICE

CUADRO A.1

Brasil: regresiones de salarios para varonesy mujeres (ecuación 10)

\begin{tabular}{|c|c|c|c|c|c|c|}
\hline & \multicolumn{3}{|c|}{ Varones } & \multicolumn{3}{|c|}{ Mujeres } \\
\hline & Coeficiente & $\begin{array}{l}\text { Desviación } \\
\text { estándar }\end{array}$ & $\mathrm{P}>|\mathrm{t}|$ & Coeficiente & $\begin{array}{l}\text { Desviación } \\
\text { estándar }\end{array}$ & $\mathrm{P}>|\mathrm{t}|$ \\
\hline Jefe & 0,9470 & 0,0465 & 0,000 & 0,0922 & 0,0567 & 0,104 \\
\hline Edad & 0,0619 & 0,0013 & 0,000 & 0,0316 & 0,0023 & 0,000 \\
\hline Edad $^{2}$ & $-0,0006$ & 0,0000 & 0,000 & $-0,0004$ & 0,0000 & 0,000 \\
\hline \multicolumn{7}{|l|}{ Blancos (referencia) } \\
\hline Negros & $-0,3251$ & 0,0081 & 0,000 & $-0,0811$ & 0,0258 & 0,002 \\
\hline Pardos & $-0,2106$ & 0,0055 & 0,000 & $-0,1085$ & 0,0139 & 0,000 \\
\hline Educación & 0,1455 & 0,0021 & 0,000 & 0,1215 & 0,0074 & 0,000 \\
\hline Sindicato & 0,2126 & 0,0045 & 0,000 & 0,2591 & 0,0059 & 0,000 \\
\hline \multicolumn{7}{|c|}{ Sin carteira (referencia) } \\
\hline Con carteira & 0,1323 & 0,0056 & 0,000 & 0,0954 & 0,0055 & 0,000 \\
\hline Funcionario público & 0,2355 & 0,0093 & 0,000 & 0,1921 & 0,0087 & 0,000 \\
\hline Autónomo & 0,2897 & 0,0063 & 0,000 & 0,2579 & 0,0065 & 0,000 \\
\hline \multicolumn{7}{|l|}{ Industria (referencia) } \\
\hline Comercio & $-0,1811$ & 0,0058 & 0,000 & $-0,0639$ & 0,0080 & 0,000 \\
\hline Servicios & $-0,0986$ & 0,0047 & 0,000 & 0,0014 & 0,0066 & 0,827 \\
\hline Otras actividades & 0,0201 & 0,0076 & 0,008 & 0,2900 & 0,0099 & 0,000 \\
\hline \multicolumn{7}{|l|}{ São Paulo (referencia) } \\
\hline Recife & $-0,6203$ & 0,0112 & 0,000 & $-0,6215$ & 0,0208 & 0,000 \\
\hline Salvador & $-0,5204$ & 0,0108 & 0,000 & $-0,4383$ & 0,0130 & 0,000 \\
\hline Belo Horizonte & $-0,2428$ & 0,0065 & 0,000 & $-0,3234$ & 0,0095 & 0,000 \\
\hline Río de Janeiro & $-0,2683$ & 0,0085 & 0,000 & $-0,2911$ & 0,0132 & 0,000 \\
\hline Porto Alegre & $-0,2228$ & 0,0065 & 0,000 & $-0,2617$ & 0,0172 & 0,000 \\
\hline \multicolumn{7}{|l|}{ Año 1992 (referencia) } \\
\hline Año 1993 & $-0,0657$ & 0,0075 & 0,000 & $-0,0715$ & 0,0089 & 0,000 \\
\hline Año 1995 & 0,1849 & 0,0076 & 0,000 & 0,1359 & 0,0113 & 0,000 \\
\hline Año 1996 & 0,1399 & 0,0078 & 0,000 & 0,2032 & 0,0092 & 0,000 \\
\hline Año 1997 & 0,1121 & 0,0078 & 0,000 & 0,2474 & 0,0098 & 0,000 \\
\hline Año 1998 & 0,0107 & 0,0094 & 0,255 & 0,2836 & 0,0117 & 0,000 \\
\hline Año 1999 & $-0,1146$ & 0,0100 & 0,000 & 0,2126 & 0,0130 & 0,000 \\
\hline Año 2001 & $-0,1066$ & 0,0091 & 0,000 & 0,1545 & 0,0123 & 0,000 \\
\hline $\mathrm{m}_{0}$ & $-0,6001$ & 0,2054 & 0,003 & 1,6072 & 0,2607 & 0,000 \\
\hline $\mathrm{m}_{1}$ & $-3,1367$ & 0,1696 & 0,000 & 3,3450 & 0,1910 & 0,000 \\
\hline $\mathrm{m}_{2}$ & 0,6079 & 0,0735 & 0,000 & 1,0418 & 0,0635 & 0,000 \\
\hline Constante & $-3,2869$ & 0,0763 & 0,000 & $-1,0634$ & 0,2242 & 0,000 \\
\hline $\mathrm{R}^{2}$ & 0,4886 & & & 0,4771 & & \\
\hline $\mathrm{N}^{\mathrm{o}}$ de observaciones & 148508 & & & 117582 & & \\
\hline
\end{tabular}


Brasil: líneas regionales de pobreza

(Dólares)

\begin{tabular}{|c|c|c|c|c|c|c|c|}
\hline Año & Recife & Salvador & Belo Horizonte & Río de Janeiro & São Paulo & Porto Alegre & Media \\
\hline 1992 & 38,6 & 36,1 & 47,1 & 48,9 & 51,4 & 55,1 & 46,2 \\
\hline 1993 & 38,3 & 37,9 & 44,1 & 43,1 & 46,3 & 49,2 & 43,2 \\
\hline 1995 & 43,6 & 43,8 & 46,8 & 47,4 & 53,4 & 49,4 & 47,4 \\
\hline 1996 & 42,2 & 41,1 & 47,9 & 46,9 & 54,0 & 49,2 & 46,9 \\
\hline 1997 & 38,9 & 36,0 & 45,1 & 46,0 & 48,7 & 47,8 & 43,8 \\
\hline 1998 & 42,2 & 40,3 & 47,0 & 47,9 & 52,1 & 51,8 & 46,9 \\
\hline 1999 & 41,5 & 40,0 & 47,3 & 47,3 & 52,7 & 49,8 & 46,4 \\
\hline 2001 & 38,6 & 38,5 & 48,8 & 50,7 & 53,0 & 54,4 & 47,3 \\
\hline Promedio & 40,5 & 39,2 & 46,8 & 47,3 & 51,4 & 50,8 & 46,0 \\
\hline
\end{tabular}

CUADRO A. 3

Brasil: personas pobres, por regiones metropolitanas

(Porcentajes)

\begin{tabular}{|c|c|c|c|c|c|c|c|c|c|c|c|c|}
\hline \multirow[b]{2}{*}{ Año } & \multicolumn{2}{|c|}{ Recife } & \multicolumn{2}{|c|}{ Salvador } & \multicolumn{2}{|c|}{ Belo Horizonte } & \multicolumn{2}{|c|}{ Río de Janeiro } & \multicolumn{2}{|c|}{ São Paulo } & \multicolumn{2}{|c|}{ Porto Alegre } \\
\hline & $\begin{array}{l}\text { Escenario } \\
\text { estimado }\end{array}$ & $\begin{array}{l}\text { Contra- } \\
\text { factual }\end{array}$ & Estimado & $\begin{array}{l}\text { Contra- } \\
\text { factual }\end{array}$ & Estimado & $\begin{array}{l}\text { Contra- } \\
\text { factual }\end{array}$ & Estimado & $\begin{array}{l}\text { Contra- } \\
\text { factual }\end{array}$ & Estimado & $\begin{array}{l}\text { Contra- } \\
\text { factual }\end{array}$ & Estimado & $\begin{array}{l}\text { Contra- } \\
\text { factual }\end{array}$ \\
\hline 1992 & 50,7 & 47,1 & 45,9 & 42,3 & 34,6 & 31,5 & 22,6 & 20,7 & 19,1 & 16,8 & 21,6 & 19,8 \\
\hline 1993 & 52,0 & 47,9 & 42,3 & 38,8 & 34,7 & 31,3 & 23,0 & 20,8 & 16,9 & 14,6 & 20,9 & 18,7 \\
\hline 1995 & 43,5 & 40,0 & 36,2 & 32,7 & 25,8 & 23,5 & 18,1 & 16,3 & 12,5 & 10,8 & 17,2 & 15,7 \\
\hline 1996 & 40,0 & 37,0 & 37,6 & 34,1 & 26,8 & 23,1 & 17,2 & 15,5 & 10,7 & 9,3 & 16,7 & 15,0 \\
\hline 1997 & 38,8 & 34,6 & 32,4 & 28,7 & 22,4 & 19,4 & 15,4 & 13,7 & 10,8 & 9,0 & 15,8 & 13,8 \\
\hline 1998 & 42,9 & 39,3 & 33,7 & 29,9 & 27,4 & 23,4 & 18,3 & 16,6 & 12,9 & 11,4 & 18,4 & 16,7 \\
\hline 1999 & 43,5 & 39,8 & 36,3 & 31,9 & 27,9 & 24,8 & 18,8 & 17,4 & 15,0 & 12,9 & 19,1 & 17,2 \\
\hline 2001 & 41,8 & 37,9 & 38,3 & 33,3 & 24,3 & 21,9 & 21,5 & 20,1 & 15,2 & 13,6 & 18,4 & 17,0 \\
\hline Promedio & 44,1 & 40,5 & 37,8 & 34,0 & 28,0 & 24,9 & 19,3 & 17,6 & 14,1 & 12,3 & 18,5 & 16,7 \\
\hline
\end{tabular}

Bibliografía

Arriagada, I. (2005): Dimensiones de la pobreza y políticas desde una perspectiva de género, Revista de la CEPAL, N 85 , LC/G.2266-P, Santiago de Chile.

Bardhan, P.K. y C. Udry (1999): Development Microeconomics, Nueva York, Oxford University Press.

Barros, R.P. y R. Mendonça (1997): O impacto do crescimento econômico e de reduções no grau de desigualdade sobre a pobreza, Texto para discussão, № 528 , Río de Janeiro, Instituto de Investigación Económica Aplicada (IPEA).

Barros, R.P., R. Henriques y R. Mendonça (2001): A estabilidade inaceitável: desigualdade e pobreza no Brasil, Texto para discussão, $\mathrm{N}^{\circ}$ 528, Río de Janeiro, Instituto de Investigación Económica Aplicada (IPEA).

Blinder, A.S. (1973): Wage discrimination: reduced form and structural estimates, Journal of Human Resources, vol. 8, № 4, Madison, Wisconsin, University of Wisconsin Press.

Bourguignon, F., F. Ferreira y N. Lustig (1998): The Microeconomics of Income Distribution Dynamics in East Asia and Latin America, Washington, D.C., Banco Mundial.
Bourguignon, F., M. Fournier y M. Gurgand (2007): Selection bias corrections based on the multinomial logit model: Monte Carlo comparisons, Journal of Economic Surveys, vol. 21, N ${ }^{\circ} 1$, Oxford, Reino Unido, Blackwell Publishing.

CEPAL (Comisión Económica para América Latina y el Caribe) (2005): Panorama social de América Latina, LC/G.2288-P, Santiago de Chile. Publicación de las Naciones Unidas, $\mathrm{N}^{\circ}$ de venta: S.05.II.G.161.

Corseuil, C.H. y M.N. Foguel (2002): Uma sugestão de deflatores para rendas obtidas a partir de algumas pesquisas domiciliares do IBCE, Texto para discussão, $N^{\circ}$ 897, Río de Janeiro, Instituto de Investigación Económica Aplicada (IPEA).

Costa, J.S., L. Pinheiros y otros (2005): A face feminina da pobreza: representação e feminização da pobreza no Brasil, Texto para discussão, No 1137, Río de Janeiro, Instituto de Investigación Económica Aplicada (IPEA).

DIEESE (Departamento Intersindical de Estadística y Estudios Socioeconómicos) (1993): Cesta básica nacional: metodologia, São Paulo. 
Gradín, C., C. Río y O. Cantó (2006): Poverty and Women's Labor Market Activity: the Role of Gender Wage Discrimination in the EU, Working Paper Series, $\mathrm{N}^{\circ}$ 2006-40, Palma de Mallorca, Society for the Study of Inequality (ECINEQ).

Heckman, J.J. (1979): Sample selection bias as a specification error, Econometrica, vol. 47, № 1, Nueva York, Econometric Society.

Hoffmann, R. (2000): Mensuração da desigualdade e pobreza no Brasil, en R. Henriques (org.), Desigualdade e pobreza no Brasil, Río de Janeiro, Instituto de Investigación Económica Aplicada (IPEA).

Hoffmann, R. y E.T. Leone (2004): Participação da mulher no mercado de trabalho e desigualdade da renda domiciliar per capita no Brasil: 1981-2002, Nova economia, vol. 14, No 2, Minas Gerais, Universidad Federal de Minas Gerais, mayo-agosto.

Juhn, C., K.M. Murphy y B. Pierce (1993): Wage inequality and rise in returns to skill, Journal of Political Economy, vol. 101, № 3 , Chicago, University of Chicago Press.

Kabeer, N. (1998): Tácticas y compromisos: nexos entre género y pobreza, en I. Arriagada y C. Torres (comps.), Género y pobreza. Nuevas dimensiones, serie Ediciones de las mujeres, $\mathrm{N}^{\circ} 26$, Santiago de Chile, ISIs Internacional.

Montaño, S. (2003): Políticas de empoderamiento de las mujeres como estrategia de lucha contra la pobreza, en R. Atria, M. Siles y otros, Capital social y reducción de la pobreza en América Latina y el Caribe: en busca de un nuevo paradigma, LC/G.2194-P, Santiago de Chile, Comisión Económica para América Latina y el Caribe (CEPAL). Publicación de las Naciones Unidas, $\mathrm{N}^{\mathrm{o}}$ de venta: S.03.II.G.03.

Oakley, A. (1972): Sex, Gender and Society: Towards a New Society, Londres, Maurice Temple Smith Ltd.

Oaxaca, R.L. (1973): Male-female differentials in urban labor market, International Economic Review, vol. 14, No 3, Oxford, Reino Unido, Blackwell Publishing.

Rocha, S. (2000a): Opções metodológicas para a estimação de linhas de indigência e de pobreza no Brasil, Texto para discussão; $\mathrm{N}^{\circ} 720$, Río de Janeiro, Instituto de Investigación Económica Aplicada.

(2000b): Pobreza e desigualdade no Brasil: o esgotamento dos efeitos distributivos do Plano Real, Texto para discussão, $\mathrm{N}^{\mathrm{o}} 721$, Río de Janeiro, Instituto de Investigación Económica Aplicada.

Sobrinho, N.F.S. (2001): Desigualdade e pobreza: fatos estilizados e simulações, Revista paranaense de desenvolvimento, $\mathrm{N}^{\circ} 100$ Curitiba, Instituto Paranaense de Desarrollo Económico y Social (IPARDES), enero-junio.

Valenzuela, M.E. (2003): Desigualdad de género y pobreza en América Latina: separata de Mujeres, pobreza y mercado de trabajo: Argentina y Paraguay, Santiago de Chile, Organización Internacional del Trabajo (OIT).

Yáñez, A.W.E. (2004): Qué explica la desigualdad en la distribución del ingreso en las áreas urbanas de Bolivia: un análisis a partir de un modelo de microsimulación, Barcelona, Universidad Autónoma de Barcelona. 